\title{
Impact of dye interlayer on the performance of organic photovoltaic devices
}

\author{
S. L. Lai, ${ }^{1}$ M. F. Lo, ${ }^{1}$ M. Y. Chan, ${ }^{2, a)}$ C. S. Lee, ${ }^{1, a)}$ and S. T. Lee ${ }^{1}$ \\ ${ }^{1}$ Center of Super-Diamond and Advanced Films (COSDAF) and Department of Physics and Materials \\ Science, City University of Hong Kong, Hong Kong SAR, China \\ ${ }^{2}$ Department of Chemistry, The University of Hong Kong, Pokfulam Road, Hong Kong SAR, China
}

(Received 28 July 2009; accepted 15 September 2009; published online 13 October 2009)

\begin{abstract}
The influences of buffer interlayer at the donor/acceptor interface on the open circuit voltage $\left(V_{\mathrm{OC}}\right)$ of typical copper phthalocyanine $(\mathrm{CuPc}) / \mathrm{C}_{60}$ organic photovoltaic devices are studied. Six fluorescent dyes with progressively increasing ionization potentials $\left(I_{P}\right)$ were used to investigate the factors influencing the $V_{\mathrm{OC}}$. The short-circuit current and fill factor of $\mathrm{CuPc} / \mathrm{C}_{60}$ device incorporating dye interlayer are lower than those of standard bilayer device. On the other hand, the $V_{\mathrm{OC}}$ increases linearly with the $I_{P}$ of dye material and falls off when the $I_{P}$ is equal to or greater than $5.6 \mathrm{eV}$, in which the energy offset between the highest occupied molecular orbitals at the interlayer/ $\mathrm{C}_{60}$ heterojunction is smaller than the $\mathrm{C}_{60}$ exciton binding energy. The findings underscore the importance of energy offsets in photovoltaic responses. (C) 2009 American Institute of Physics. [doi:10.1063/1.3243991]
\end{abstract}

It is well known that the short-circuit current density $\left(J_{\mathrm{SC}}\right)$ strongly depends on the absorption efficiency and the exciton diffusion length in the photoactive materials, while the open-circuit voltage $\left(V_{\mathrm{OC}}\right)$ is predominately determined by the energy difference between the highest occupied molecular orbital (HOMO) of the donor (D) and the lowest unoccupied molecular orbital (LUMO) of the acceptor (A), i.e., $\mathrm{HOMO}_{\mathrm{D}}-\mathrm{LUMO}_{\mathrm{A}} \cdot{ }^{1-6}$

Currently, most organic photovoltaic (OPV) devices employ two photoactive materials to form the donor-acceptor junction where photogenerated exciton dissociation and charge transfer take place. To further improve the photovoltaic responses, the use of more complicated device structures with multiple photoactive layers is one feasible approach. Sista et al. ${ }^{7}$ recently reported three-component OPV devices with a cascade-type energy level structure, in which a thin interfacial layer, copper phthalocyanine ( $\mathrm{CuPc})$, was inserted between the donor/acceptor interface. The requirements for such a cascade-type energy band structure is that the ionization potential $\left(I_{P}\right)$ of the interlayer should be larger than that of $p$-type material (donor) while its electron affinity $\left(E_{A}\right)$ should be smaller than that of $n$-type material (acceptor). This approach can effectively engineer the $V_{\mathrm{OC}}$ of OPV devices via choosing interlayers with appropriate energy levels. Kinoshita et $a l^{8}{ }^{8}$ have also demonstrated the use of a thin zinc phthalocyanine interfacial layer $(\sim 2 \mathrm{~nm})$ between pentacene and $\mathrm{C}_{60}$ to improve both $J_{\mathrm{SC}}$ and $V_{\mathrm{OC}}$. On the other hand, Rand et al. ${ }^{9}$ reported that the use of an interfacial layer would negatively affect the $J_{\mathrm{SC}}$ if the interfacial layer thickness exceeds $5 \mathrm{~nm}$. While the cascade-type energy band structure provides a viable means to improve the photovoltaic response, there is little information on how the device performance is related to the constituent materials. In order to optimize the performance of such three-component OPV devices, systematic studies on the influence of the interlayer would obviously be desirable. In this work, we report the use

\footnotetext{
a) Authors to whom correspondence should be addressed. Electronic addresses: chanmym@hku.hk and apcslee@cityu.edu.hk.
}

of six representative fluorescent dyes as interfacial layers to form the cascade-type energy band structure, from which we determine the ideal material parameters for the threecomponent OPV devices. In addition, the relation between the $V_{\mathrm{OC}}$ and the offset energies at the donor-acceptor interface is discussed.

Six representative fluorescent dyes, namely 4-(dicyanomethylene)-2-tert-butyl-6-(1,1,7,7-tetramethyljulolidyl-9-enyl)4H-pyran (DCJTB), 4-(dicyanomethylene)-2-tert-butyl(1,3,3,7,7,-penta-methyljulolidyl-9-enyl)-4H-pyran (DCJPB), tetra(t-butyl)rubrene (TBRB), rubrene (RB), 10-(2-benzothiazolyl)-1,1,7,7-tetramethyl-,3,6,7-tetrahydro- $1 \mathrm{H}, 5 \mathrm{H}, 11 \mathrm{H}$ benzo[l] pyrano[6 7 8-ij] quinolizin-11-one (C545T), and 4,4'-bis(2,2'-diphenyl vinyl)-1, 1' '-biphenyl (DPVBi), were inserted as an interlayer between $\mathrm{CuPc}$ (donor) and $\mathrm{C}_{60}$ (acceptor). These six materials were selected because of their progressively increasing ionization potentials $\left(I_{P}\right)$ that allow us to study the dependence of $V_{\mathrm{OC}}$ on the offset energies at the organic heterojunctions. ${ }^{10-15}$ For comparison, a standard bilayer device with the structure of $\mathrm{ITO} / \mathrm{CuPc} / \mathrm{C}_{60} /$ bathocuproine (BCP)/Al was also fabricated. Currentvoltage characteristics of OPV devices were measured with a programmable Keithley model 237 power source. The photocurrent was measured in the dark and under illumination with an intensity of $100 \mathrm{~mW} / \mathrm{cm}^{2}$ from an Oriel $150 \mathrm{~W}$ solar simulator equipped with AM1.5G (AM: air mass, G: global) filters, and the light intensity was measured with a calibrated silicon detector with a KG-5 color filter. ${ }^{16}$ Absorption spectra of organic films on quartz substrates were measured with a Perkin Elmer Lambda 2S UV/VIS spectrometer.

Table I summarizes the key cell parameters for devices with the structure of ITO/CuPc $(34 \mathrm{~nm}) /$ dye $(5 \mathrm{~nm}) / \mathrm{C}_{60}(40$ $\mathrm{nm}) / \mathrm{BCP}(5 \mathrm{~nm}) / \mathrm{Al}(80 \mathrm{~nm})$, in which DCJTB, DCJPB, TBRB, RB, C545T, and DPVBi were used as dye interlayer, respectively, together with the standard device. Under illumination, the standard device exhibits a $J_{\mathrm{SC}}$ of $6.40 \mathrm{~mA} / \mathrm{cm}^{2}$, a $V_{\mathrm{OC}}$ of $0.46 \mathrm{~V}$, a fill factor $(\mathrm{FF})$ of 0.52 , and a power conversion efficiency $\left(\eta_{P}\right)$ of $1.54 \%$. However, the $J_{\mathrm{SC}}$ drops sharply when a thin dye interlayer is inserted between $\mathrm{CuPc}$ 
TABLE I. Key photovoltaic responses for devices without and with different dye interlayers.

\begin{tabular}{lcccc}
\hline \hline Dye & $\begin{array}{c}J_{\mathrm{SC}} \\
\left(\mathrm{mA} / \mathrm{cm}^{2}\right)\end{array}$ & $\begin{array}{c}V_{\mathrm{OC}} \\
(\mathrm{V})\end{array}$ & FF & $\begin{array}{c}\eta_{P} \\
(\%)\end{array}$ \\
\hline Standard & 6.40 & 0.46 & 0.52 & 1.54 \\
DCJTB & 2.48 & 0.51 & 0.42 & 0.53 \\
DCJPB & 3.41 & 0.53 & 0.43 & 0.76 \\
TBRB & 2.90 & 0.60 & 0.39 & 0.67 \\
RB & 3.47 & 0.62 & 0.39 & 0.83 \\
C5454T & 3.98 & 0.48 & 0.45 & 0.86 \\
DPVBi & 0.67 & 0.54 & 0.44 & 0.16 \\
\hline \hline
\end{tabular}

and $\mathrm{C}_{60}$. The poor $J_{\mathrm{SC}}$ may be attributed to the absence of $\mathrm{C}_{60}$ exciton dissociation (see discussion below) and the relatively low absorption coefficients of dye materials. In particular, the absorption coefficients of all dye materials are on the order of $10^{4} \mathrm{~cm}^{-1}$, which are one order of magnitude lower than those of $\mathrm{CuPc}$ and $\mathrm{C}_{60} \cdot{ }^{17}$ The low absorption would inevitably lead to a smaller number of photogenerated excitons, and thus a lower photocurrent. The low photocurrent may also be attributed to the relatively low carrier mobility in dye materials. Particularly, carrier mobilities in dye materials (on the order of $10^{-5}-10^{-7} \mathrm{~cm}^{2} \mathrm{~V}^{-1} \mathrm{~s}^{-1}$ ) are comparatively smaller than those in $\mathrm{CuPc}$ and $\mathrm{C}_{60}$, provided that the hole mobility in $\mathrm{CuPc}$ is on the order of $10^{-4} \mathrm{~cm}^{2} \mathrm{~V}^{-1} \mathrm{~s}^{-1}$ and the electron mobility in $\mathrm{C}_{60}$ is on the order of $10^{-2} \mathrm{~cm}^{2} \mathrm{~V}^{-1} \mathrm{~s}^{-1}$. The low carrier mobility would impede the charge carrier transport to the respective electrodes. It should be mentioned that the $J_{\mathrm{SC}}$ for the DPVBibased device is exceptionally low $\left(0.67 \mathrm{~mA} / \mathrm{cm}^{2}\right)$, about one-fourth that of the devices with other dyes. One reason for the low $J_{\mathrm{SC}}$ is the extremely low absorption of DPVBi in the visible spectrum. Figure 1 depicts the normalized absorption spectra of the thin films of the dye materials on quartz substrates. Clearly, DPVBi absorbs light in the UV region that coincides with the absorption cutoff of the ITO coated glass substrate, i.e., wavelength $\lambda \leq 360 \mathrm{~nm}$. This definitely limits photon harvest in the DPVBi layer.

On the other hand, the $V_{\mathrm{OC}}$ increases from 0.46 to $0.62 \mathrm{~V}$ and then falls off to $0.48 \mathrm{~V}$ when a C545T interlayer is used. To investigate the controlling factors of $V_{\mathrm{OC}}$ in the three-

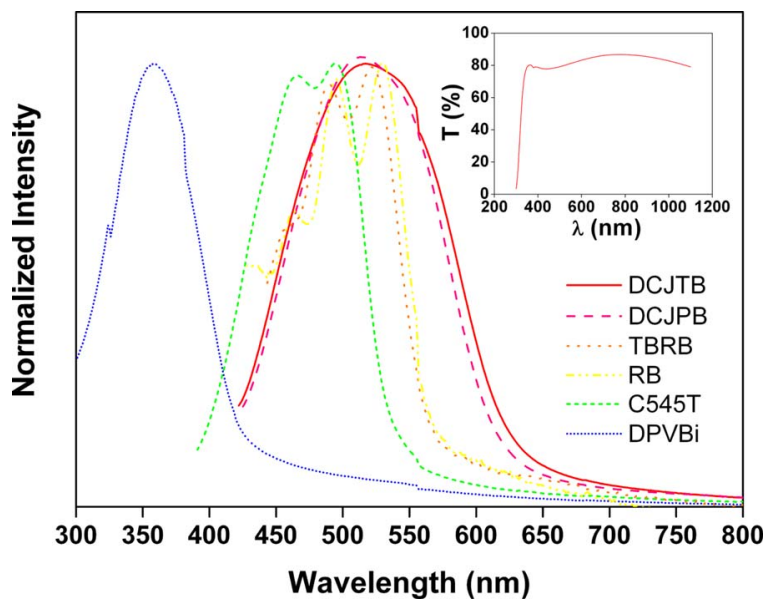

FIG. 1. (Color online) Normalized absorption spectra of thin films of dye materials on quartz substrates. Inset: Transmission of ITO coated glass substrate with reference to air.

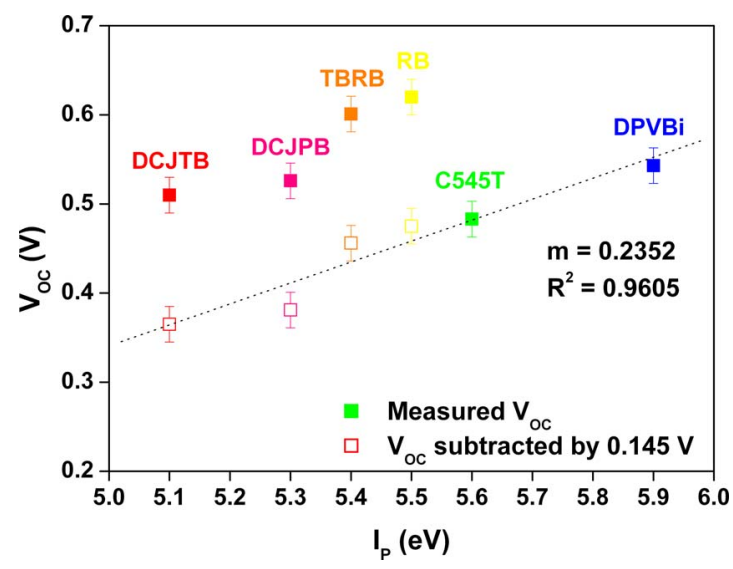

FIG. 2. (Color online) $V_{\mathrm{OC}}$ of devices with different dye interlayers as a function of $I_{P}$ of dye materials.

component OPV devices, the $V_{\mathrm{OC}}$ of devices are plotted as a function of $I_{P}$ of the dyes in Fig. 2. The $V_{\mathrm{OC}}$ of a device with an interlayer of smaller $I_{P}$ (i.e., $I_{P} \leq 5.5 \mathrm{eV}$ ) is generally larger than that of a device with an interlayer having $I_{P}$ equal or larger than $5.6 \mathrm{eV}$. In addition, by subtracting the $V_{\mathrm{OC}}$ of the former devices by $0.145 \mathrm{~V}$, a linear fit to the data with a slope of 0.24 and a correlation coefficient $\left(R^{2}\right)$ of 0.96 can be drawn. In particular, the line represents a linear relation between the $V_{\mathrm{OC}}$ and the $I_{P}$ of dye materials. It is interesting to note that the linear fit intercepts the $x$-axis at $3.6 \mathrm{eV}$, which corresponds to the LUMO level of $\mathrm{C}_{60}$ measured by inverse photoelectron spectroscopy. ${ }^{18}$ This is in good agreement with an increase in $V_{\mathrm{OC}}$ with increasing the energy difference, that is, HOMO (dye)-LUMO $\left(\mathrm{C}_{60}\right)$ as derived.

The voltage difference of $0.145 \mathrm{~V}$ may be attributed to the $V_{\mathrm{OC}}$ contribution from the presence of $\mathrm{C}_{60}$ exciton dissociation. Table II lists the energy levels of organic materials. All energy levels of organic materials are taken from the literatures, ${ }^{10-15,18}$ in which the LUMO levels of CuPc and $\mathrm{C}_{60}$ were measured by inverse photoelectron spectroscopy, so called "true LUMO", and those of dye materials were estimated from the optical absorption spectra, referred as "optical LUMO", in which the exciton binding energies $\left(E_{B}\right)$ of the dye materials have not been considered. $E_{B}$ is the minimum energy needed to overcome the Coulomb electric force to separate the exciton into free electron and hole, and is given by the energy difference between the true LUMO and the optical LUMO, i.e., $E_{B}=E_{\text {true LUMO }}-E_{\text {optical LUMo. Dju- }}$ rovich et al. ${ }^{18}$ recently reported that $E_{B}$ in most molecular organic semiconductors linearly increases with increasing energy gap (either transport gap, $E_{T}$, i.e., $E_{T}=E_{\mathrm{HOMO}}$

TABLE II. Energy levels of dye materials used. $E_{\text {opt }}, E_{T}$, and $E_{B}$ are the optical bandgap, the transport gap, and the exciton binding energy in the organic materials, respectively. The electron affinities of $\mathrm{CuPc}$ and $\mathrm{C}_{60}$ were measured by the inverse photoelectron spectroscopy, in which $E_{\text {true LuMO }}$ levels were directly determined.

\begin{tabular}{lcccccccc}
\hline \hline Material & CuPc & DCJTB & DCJPB & TBRB & RB & C545T & DPVBi & $\mathrm{C}_{60}$ \\
\hline$E_{A}(\mathrm{eV})$ & 3.2 & 3.1 & 3.2 & 3.2 & 3.3 & 2.8 & 2.8 & 3.5 \\
$I_{P}(\mathrm{eV})$ & 5.2 & 5.1 & 5.3 & 5.4 & 5.5 & 5.6 & 5.9 & 6.2 \\
$E_{\text {opt }}(\mathrm{eV})$ & $\cdots$ & 2.0 & 2.1 & 2.2 & 2.2 & 2.8 & 3.1 & $\ldots$ \\
$E_{T}(\mathrm{eV})$ & 2.0 & 2.3 & 2.4 & 2.6 & 2.6 & 3.4 & 3.9 & 2.7 \\
$E_{B}(\mathrm{eV})$ & 0.3 & 0.3 & 0.3 & 0.4 & 0.4 & 0.6 & 0.8 & 0.7 \\
\hline \hline
\end{tabular}


TABLE III. Conditions for exciton dissociation at the organic/organic interfaces, given that $E_{B}$ of $\mathrm{CuPc}$ and $\mathrm{C}_{60}$ are 0.3 and $0.7 \mathrm{eV}$, respectively. $\Delta E_{\mathrm{LUMO}}$ is calculated from the difference between the $E_{\text {true LUMO }}(\mathrm{CuPc}$ or $\mathrm{C}_{60}$ ) and $E_{\text {true Lumo }}$ (dye), in which $E_{\text {true LuMO }}$ (dye) is estimated according to the equation $E_{B}=E_{\text {true LUMO }}-E_{\text {optical LUMO }} \checkmark \checkmark$ and $x$ show whether excitons can or cannot be dissociated in the particular layers at various interfaces.

\begin{tabular}{|c|c|c|c|c|c|}
\hline Interface & $\Delta E_{\mathrm{LUMO}}$ & $\Delta E_{\text {Номо }}$ & $\mathrm{CuPc}$ & Dye & $\mathrm{C}_{60}$ \\
\hline $\mathrm{CuPc} / \mathrm{C}_{60}$ & 0.3 & 1.0 & $\checkmark$ & $\cdots$ & $\checkmark$ \\
\hline $\mathrm{CuPc/DCJTB}$ & 0.4 & 0.1 & $\checkmark$ & $x$ & $\cdots$ \\
\hline $\mathrm{CuPc} / \mathrm{DCJPB}$ & 0.3 & 0.1 & $\checkmark$ & $x$ & $\cdots$ \\
\hline $\mathrm{CuPc} / \mathrm{TBRB}$ & 0.4 & 0.2 & $\checkmark$ & $x$ & $\cdots$ \\
\hline $\mathrm{CuPc} / \mathrm{RB}$ & 0.3 & 0.3 & $\checkmark$ & $x$ & $\cdots$ \\
\hline $\mathrm{CuPc} / \mathrm{C} 545 \mathrm{~T}$ & 1.0 & 0.4 & $\checkmark$ & $x$ & $\cdots$ \\
\hline $\mathrm{CuPc} / \mathrm{DPVBi}$ & 1.2 & 0.7 & $\checkmark$ & $x$ & $\cdots$ \\
\hline $\mathrm{DCJTB} / \mathrm{C}_{60}$ & 0.7 & 1.1 & $\cdots$ & $\checkmark$ & $\checkmark$ \\
\hline $\mathrm{DCJPB} / \mathrm{C}_{60}$ & 0.6 & 0.9 & $\cdots$ & $\checkmark$ & $\checkmark$ \\
\hline $\mathrm{TBRB} / \mathrm{C}_{60}$ & 0.7 & 0.8 & $\cdots$ & $\checkmark$ & $\checkmark$ \\
\hline $\mathrm{RB} / \mathrm{C}_{60}$ & 0.6 & 0.7 & $\cdots$ & $\checkmark$ & $\checkmark$ \\
\hline $\mathrm{C} 545 \mathrm{~T} / \mathrm{C}_{60}$ & 1.3 & 0.6 & $\cdots$ & $\checkmark$ & $x$ \\
\hline $\mathrm{DPVBi} / \mathrm{C}_{60}$ & 1.5 & 0.3 & $\cdots$ & $\checkmark$ & $x$ \\
\hline
\end{tabular}

- $E_{\text {true LUMO }}$, or optical bandgap, $E_{\text {opt }}$, i.e., $E_{\text {opt }}=E_{\mathrm{HOMO}}$

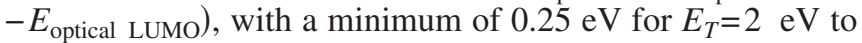
$1.5 \mathrm{eV}$ for $E_{T}=6 \mathrm{eV}$.

For efficient exciton dissociation into free charge carriers at the donor/acceptor interface, the energy level offset for the HOMO or LUMO ( $\Delta E_{\mathrm{HOMO}}$ or $\Delta E_{\mathrm{LUMO}}$, respectively) should be larger than $E_{B}{ }^{19,20}$ In particular, $\Delta E_{\mathrm{HOMO}}$ $\left(\Delta E_{\mathrm{LUMO}}\right)$ should be larger than the acceptor (donor) exciton binding energy for efficient exciton dissociation in the acceptor (donor). ${ }^{20}$ In other words, excitons in $\mathrm{C}_{60}$ would be dissociated if the $\Delta E_{\mathrm{HOMO}}$ at the interlayer/ $\mathrm{C}_{60}$ interface is larger than the $E_{B}$ in $\mathrm{C}_{60}(0.7 \mathrm{eV})$; while excitons in $\mathrm{CuPc}$ only dissociate if the $\Delta E_{\mathrm{LUMO}}$ at the CuPc/interlayer interface is larger than $0.3 \mathrm{eV} .{ }^{18}$ At the same time, excitons in dye material would be dissociated if the $\Delta E_{\mathrm{HOMO}}$ at the $\mathrm{CuPc} /$ interlayer and $\Delta E_{\mathrm{LUMO}}$ at the interlayer/C $\mathrm{C}_{60}$ interface, respectively, are larger than the $E_{B}$ in dye material. Table III summarizes the conditions for exciton dissociation at different organic/organic interfaces, in which the $\Delta E_{\mathrm{LUMO}}$ is calculated from the difference between the $E_{\text {true LuMO }}(\mathrm{CuPc}$ or $\mathrm{C}_{60}$ ) and $E_{\text {true Lumo }}(\mathrm{dye})$, as shown in Table II. At the interlayer/ $\mathrm{C}_{60}$ interfaces, efficient $\mathrm{C}_{60}$ exciton dissociation takes place at the interfaces between the dye materials and $\mathrm{C}_{60}$. Except at the $\mathrm{C} 545 \mathrm{~T} / \mathrm{C}_{60}$ and $\mathrm{DPVBi} / \mathrm{C}_{60}$ contacts, the close proximity of the HOMO levels of $\mathrm{C}_{60}$ and dye materials prohibits $\mathrm{C}_{60}$ exciton dissociation; particularly the $\Delta E_{\mathrm{HOMO}}$ is much smaller than the exciton binding energy in $\mathrm{C}_{60}\left(E_{B}=0.7 \mathrm{eV}\right)$. On the other hand, all excitons in the interlayer are expected to dissociate at the interlayer/ $\mathrm{C}_{60}$ interfaces [i.e., $\Delta E_{\mathrm{LUMO}} \geq E_{B}($ dye $)$ ], especially when the $E_{B}$ in dye material has been counted. However, it is not the case at the CuPc/interlayer interfaces, in which only $\mathrm{CuPc}$ excitons can be efficiently dissociated. The close proximity of the HOMO levels of CuPc and dye materials prohibits the dye exciton dissociation; particularly the $\Delta E_{\mathrm{HOMO}}$ is much smaller than the exciton binding energy in dye materials.

As $\mathrm{C}_{60}$ exciton dissociation only takes place under certain conditions, this may account for the origin of the voltage difference between devices with interlayer having smaller $I_{P}$ (i.e., $I_{P} \leq 5.5 \mathrm{eV}$ ) and those having $I_{P}$ equal or greater than $5.6 \mathrm{eV}$. This may also explain the loss of photocurrent when a $5 \mathrm{~nm}$ thick tin(II) phthalocyanine $(\mathrm{SnPc})$ is inserted between the $\mathrm{CuPc}$ and $\mathrm{C}_{60}$ layers, as here the energy level offset is not sufficiently large for efficient $\mathrm{CuPc}$ exciton dissociation and thus results in the lower photocurrent, as reported by Rand et al. ${ }^{9}$ It is worth noting that the present results suggest that $V_{\mathrm{OC}}$ is related to exciton dissociation in both the donor and the acceptor. The absence of either donor or acceptor exciton dissociation would result in a lower $V_{\mathrm{OC}}$. The exact mechanism or physical basis for this is still unclear, and more investigation is definitely needed.

In summary, we have used fluorescent dyes as interfacial layers between $\mathrm{CuPc}$ and $\mathrm{C}_{60}$ to form the cascade-type energy level structure. With the insertion of the dye interlayer, photovoltaic responses such as $J_{\mathrm{SC}}$ and FF are reduced compared to those of the standard device. The decreased photocurrent may be attributed to the absence of $\mathrm{C}_{60}$ exciton dissociation, given that the absorption coefficients in $\mathrm{CuPc}$ and $\mathrm{C}_{60}$ are an order of magnitude higher than those in dye materials, as well as the relatively low carrier mobility in dye materials. On the other hand, the $V_{\mathrm{OC}}$ linearly increases with the $I_{P}$ of the dye interlayer, provided that the energy offset of HOMO levels at the interlayer/ $\mathrm{C}_{60}$ interfaces is larger than the $\mathrm{C}_{60}$ exciton binding energy. In particular, the $V_{\mathrm{OC}}$ falls off when the $I_{P}$ of dye materials is equal to or greater than $5.6 \mathrm{eV}$, which may be attributed to the absence of $\mathrm{C}_{60}$ exciton dissociation. Our findings provide guideline in material and device design with respect to improving the photovoltaic responses of trilayer OPV devices.

This work was supported by the Research Grants Council of Hong Kong (Project No. CityU 101707).

${ }^{1}$ P. Peumans, S. Uchida, and S. R. Forrest, Nature (London) 425, 158 (2003).

${ }^{2}$ S. Uchida, J. Xue, B. P. Rand, and S. R. Forrest, Appl. Phys. Lett. 84, 4218 (2004)

${ }^{3}$ P. Sullivan, S. Heutz, S. M. Schultes, and T. S. Jones, Appl. Phys. Lett. 84, 1210 (2004).

${ }^{4}$ T. Taima, M. Chikamatsu, Y. Yoshida, K. Saito, and K. Yase, Appl. Phys. Lett. 85, 6412 (2004).

${ }^{5}$ M. C. Scharber et al., Adv. Mater. 18, 789 (2006)

${ }^{6}$ B. P. Rand, D. P. Burk, and S. R. Forrest, Phys. Rev. B 75, 115327 (2007). ${ }^{7}$ S. Sista, Y. Yao, Y. Yang, M. L. Tang, and Z. Bao, Appl. Phys. Lett. 91, 223508 (2007).

${ }^{8}$ Y. Kinoshita, T. Hasobe, and H. Murata, Appl. Phys. Lett. 91, 083518 (2007).

${ }^{9}$ B. P. Rand, J. Xue, F. Yang, and S. R. Forrest, Appl. Phys. Lett. 87, 233508 (2005).

${ }^{10}$ Y. M. Wang, F. Teng, Z. Xu, Y. B. Hou, S. Y. Yang, and X. R. Xu, Mater. Chem. Phys. 92, 291 (2005).

${ }^{11}$ M. J. Chang, W. Y. Huang, and W. C. Huang, U.S. Patent No. 6,649,089 (18 November 2003).

${ }^{12}$ Y. S. Wu, T. H. Liu, H. H. Chen, and C. H. Chen, Thin Solid Films 496, 626 (2006).

${ }^{13}$ H. Kanno, Y. Hamada, and H. Takahashi, IEEE J. Sel. Top. Quantum Electron. 10, 30 (2004)

${ }^{14}$ K. Okumoto, H. Kanno, Y. Hamaa, H. Takahashi, and K. Shibata, Appl. Phys. Lett. 89, 063504 (2006).

${ }^{15}$ W. C. Shen, Y. K. Su, and L. W. Ji, J. Cryst. Growth 293, 48 (2006).

${ }^{16}$ V. Shrotriya, G. Li, Y. Yao, T. Moriarty, K. Emery, and Y. Yang, Adv. Funct. Mater. 16, 2016 (2006).

${ }^{17}$ P. Peumans, A. Yakimov, and S. R. Forrest, J. Appl. Phys. 93, 3693 (2003).

${ }^{18}$ P. I. Djurovich, E. I. Mayo, S. R. Forrest, and M. E. Thompson, Org. Electron. 10, 515 (2009).

${ }^{19}$ S. S. Sun, Mater. Sci. Eng., B 116, 251 (2005).

${ }^{20}$ P. Peumans and S. R. Forrest, Chem. Phys. Lett. 398, 27 (2004). 